\title{
El desarrollo agropecuario de los pueblos de alta montaña. La Peñuela, Estado de México
}

\section{The agricultural development of the high-mountain villages. La Peñuela, State of Mexico}

\author{
Nancy Diana Anastacio-Martínez* \\ Gabino Nava-Bernal * \\ Sergio Franco-MaAss*
}

\begin{abstract}
This paper analyzes the agricultural development of La Peñuela, a high-mountain village which is part of Nevado de Toluca National Park. Ethno-history was used as methodological approach which allowed us to identify the historical process of agricultural activities and their influence on other resources such as the forest-related ones. Participatory research tools, such as semi-structured interviews, were employed. The results suggest that changes in the farming systems have influenced the management of timber and non-timber resources, as well as water supply for subsistence, ever since the foundation of the community.
\end{abstract}

Keywords: Nevado de Toluca, high mountain villages, Ethno-history.

\section{Resumen}

Se analiza el desarrollo agropecuario de La Peńuela, un pueblo de alta montańa perteneciente al Parque Nacional Nevado de Toluca. El acercamiento metodológico se basó en la Etnohistoria, la cual nos permitió identificar el proceso histórico de las actividades agropecuarias y su influencia en otros recursos como los forestales. Se emplearon herramientas de investigación participativa, como las entrevistas semiestructuradas. Los resultados sugieren que los cambios en las actividades agropecuarias han influido en el manejo de los recursos forestales maderables y no maderables desde la fundación de la comunidad, así como la disponibilidad del agua para la subsistencia.

Palabras clave: Nevado de Toluca, alta montańa, etnohistoria.

* Universidad Autónoma del Estado de México. Correos-e: anancy_diana@hotmail.com, gnavab@uaemex.mx, sfrancom@uaemex.mx 


\section{Introducción}

Los pueblos de alta montaña en México han tenido un desarrollo histórico muy relacionado con su localización geográfica y con las limitaciones que imponen los territorios forestales a grandes altitudes. Se trata de localidades fundadas en zonas agrestes muy alejadas de los centros de población y de las regiones de mayor actividad económica que se establecen en condiciones ambientales poco aptas para el desarrollo de las actividades agropecuarias. Si bien es cierto que el devenir de las localidades se explica en buena medida por el discurrir histórico regional y nacional, también es cierto que dicho desarrollo depende grandemente de las condiciones locales prevalecientes.

En el Estado de México los pueblos de alta montaña se localizan en torno a las grandes elevaciones del eje neovolcánico transversal, a altitudes que rondan los 3,000 msnm (zonas de vocación forestal donde decrece significativamente la productividad agrícola), con climas semifríos y en estrecha relación con bosques de coníferas, principalmente de pino y oyamel. En este contexto, el crecimiento de las localidades ha guardado una fuerte dependencia con la extracción de recursos naturales y la apertura de zonas de pastoreo, lo que ha traído como consecuencia el deterioro de los ecosistemas forestales.

Cuando se analiza la problemática ambiental de los sistemas montañosos se puntualiza sobre los efectos negativos que tiene la presencia humana en proximidad a las zonas boscosas. El recuento de los daños incluye la deforestación, la pérdida de la biodiversidad, el deterioro de los suelos, el incremento de plagas y enfermedades, etc. Un aspecto que suele soslayarse en los estudios ambientales es que la situación que prevalece en los pueblos de alta montańa es el resultado de procesos históricos de poblamiento y apropiación del entorno geográfico (Conanp, 2013a).

El presente trabajo parte del supuesto de que cualquier planteamiento de conservación y uso racional de las montañas en México debe considerar a los habitantes de la región como agentes ambientales de cambio y que, para lograr esto, es preciso conocer los procesos históricos locales que derivaron en dicho poblamiento. En este sentido, existen pocos documentos que nos permitan conocer a mayor profundidad las particularidades de los procesos históricos locales.

En estas circunstancias se planteó la necesidad de llevar a cabo una investigación que permitiera indagar, a nivel de localidad, las particularidades del desarrollo histórico de las actividades productivas. Dada la naturaleza de la investigación se eligió para tal efecto a la comunidad de La Peñuela, ubicada en la ladera occidental del Parque Nacional Nevado de Toluca. Se trata de un pueblo con una población cercana al medio millar 
de habitantes que comparte muchas de sus características con otros asentamientos rurales en torno al volcán Xinantécatl y con localidades de otros sistemas montañosos como la Sierra de las Cruces y la Sierra Nevada.

El acercamiento metodológico utilizado aquí tiene su base en la etnohistoria, disciplina que estudia al origen de las comunidades y su convivencia con otros grupos humanos, considerando la identidad que las representa. La principal herramienta empleada para la obtención de los datos cualitativos fue la entrevista estructurada que, consiste en una guía de preguntas aplicadas a informantes clave. Dada la naturaleza de la información requerida, las entrevistas se aplicaron a personas oriundas de la localidad cuyas edades fluctuaban entre los 60 y 95 años. Dichos cuestionarios estuvieron dirigidos a conocer aspectos generales de la localidad tales como origen, fundadores, recursos extraídos y actividades económicas. La información obtenida a través de testimonios se contrastó con documentos impresos como los archivos históricos de La hacienda La Gavia e información histórica de la agricultura en el Estado de México.

En sus orígenes se trataba de una ranchería dedicada al pastoreo extensivo de ganado bovino que a lo largo de una centuria transitó lentamente hasta constituirse en una comunidad dependiente de la agricultura de riego con cultivos comerciales, principalmente de papa y flores de ornato. A lo largo de este proceso se identifican dos aspectos fundamentales: la permanente dependencia de la extracción de recursos naturales para el sostenimiento de la población y la fuerte vinculación de los productores hacia las cambiantes demandas de los mercados agropecuarios regionales. A partir de los datos históricos recabados queda claro que, a diferencia de lo ocurrido a nivel estatal, el proceso local adquiere matices particulares por la abundancia del agua de riego.

No obstante la dificultad de establecer periodos concretos fue posible identificar cinco etapas históricas recientes con características más o menos definidas. La primera etapa corresponde al establecimiento de la ranchería a principios del siglo xx para la crianza extensiva de ganado vacuno; la segunda se extiende desde inicio de los veinte hasta finales de los cuarenta en que prevalece la actividad ganadera y surge una actividad agrícola fundamentalmente de subsistencia; la tercera etapa ocurre en los años cincuenta y consiste en la transición de la actividad pecuaria hacia el desarrollo de la agricultura comercial, principalmente del cultivo de papa; la cuarta etapa se extiende desde los ańos sesenta hasta los noventa y se trata de un lento proceso de consolidación del cultivo de la papa, la tecnificación y la rotación de cultivos; la quinta y última etapa comprende la primera década del siglo Xxi y apunta hacia la transición de la localidad hacia el cultivo comercial de flores de ornato. 
El uso de la etnohistoria ha sido fundamental para recuperar y revalorizar el proceso histórico de los pueblos de alta montańa. A partir de la búsqueda de fuentes de datos y eventos en un contexto social que generó o suprimió cambios en una comunidad fue posible recuperar la realidad cotidiana de los grupos sociales que habitan la localidad de La Peńuela. En este contexto, la extracción y sobreexplotación de recursos naturales en la localidad ha estado fuertemente vinculada a los cambios históricos en las actividades productivas. Ante las difíciles condiciones que se viven en los pueblos de alta montańa, el uso del medio natural (agua y recursos forestales) se convierte en un recurso fundamental para la supervivencia de la comunidad.

\section{Antecedentes históricos: la hacienda La Gavia}

Hacia 1539, una vez consumada la conquista española, los territorios en torno al Nevado de Toluca pasaron a formar parte de la dotación de tierras que, a manera de encomienda, se otorgaron a don Alonso de Ávila, subalterno del conquistador Cortés. Dada su ubicación geográfica se trataba de territorios con una vocación agropecuaria muy limitada (HernándezRodríguez, 2005). La producción de trigo y otros granos se veía afectada por la inexistencia de mercados y las difíciles condiciones climáticas. Con el paso del tiempo la encomienda pasó a ser una gran hacienda ${ }^{1}$ dedicada primordialmente a la explotación ganadera. A pesar de su enorme riqueza forestal, los primeros encomenderos como don Juan Altamirano, don Juan Pantoja y don Francisco Chávez consideraron a estos territorios como una propiedad de poca relevancia. Hacia la segunda mitad del siglo XVI la encomienda pasó a manos de la familia Sámano, recibió el nombre hacienda de Nuestra Señora de La Candelaria, y en 1660 don Juan de Sámano Salamanca le confirió el apelativo de "Hacienda La Gavia" (Velázquez y Pérez, 1953; AGNEM, 1663; AGN, 1668).

En 1715 La Gavia fue vendida al colegio jesuita que poseía ya los territorios aledańos de Texcaltitlán (La Gavia Chica). De esta manera se conformó una gran hacienda cuya superficie superaba las 125,000 hectáreas, la mayor parte de las cuales se encontraban cubiertas por bosques templados de coníferas. La Gavia Grande tenía una extensión aproximada

${ }^{1}$ La palabra hacienda se utilizaba en la Nueva Espańa en el siglo xvi para designar una propiedad territorial de importancia que denotaba riqueza personal y que atraía población de comunidades indígenas y que mantenía servicios religiosos y aprovisionamiento seguro (Rosenzweig et al., 1987).

La formación de las haciendas en el Estado de México articulaba la metrópoli y se convirtió en la institución económica central de México, pues captaba y utilizaba el conocimiento milenario de los agricultores nativos en el manejo de las plantas, de la tierra y del agua, y empleó directa e indirectamente su fuerza de trabajo de manera casi ilimitada (Rosenzweig et al., 1987; Montes de Oca, 1998). 
de 65,000 hectáreas y la Gavia Chica abarcaba cerca de 60,000 hectáreas (Guzmán-Urbiola, 1998).

Los jesuitas poseyeron La Gavia durante cerca de cincuenta años hasta que fueron expulsados de la Nueva España en 1767. Fue este un periodo de bonanza y prosperidad en que se dinamizó la actividad agrícola y ganadera, se implementó el pago a través del sistema de raya, se adoptaron nuevas técnicas de cultivo para la producción de trigo, maíz, haba y cebada, y se incrementó la producción de ganado ovino y bovino. La crianza del ganado permitió la producción de cuero, lana, saleas y queso (Guzmán-Urbiola, 1998; Montes de Oca, 1998). Sin embargo, la iglesia tenía varias haciendas importantes en el Valle de Toluca y para 1764 los jesuitas poseían 52 haciendas, 15 ranchos y cinco trapiches azucareros. (Menegus-Bornemann, 1998; Montes de Oca, 1998).

Con la expulsión de los jesuitas La Gavia pasó a manos de la junta de temporalidades que la administró hasta 1777 , año en que fue adquirida por el primer conde de Regla, don Pedro Romero de Terreros. La hacienda mantuvo su vocación agrícola y pecuaria durante todo el siglo XIX hasta el porfiriato. Se estima que la mayor producción de cultivos se centró en el grano de maíz con 4,500 cargas, ${ }^{2}$ 1,500 cargas de trigo, 2,000 cargas de cebada y en menor cantidad el haba con 100 cargas (Villada, 1893), mientras que su producción pecuaria estuvo basada en el ganado bovino y en menor proporción en el ganado asnal (cuadro 1). Así, se producía trigo para su venta al molino La Unión, propiedad de la familia Henkel, también se criaba ganado de cerda para su venta en los mercados de Toluca y en las minas del sur del valle.

A lo largo del siglo, sin embargo, se fueron incorporando algunas actividades productivas tales como la extracción comercial de madera a gran escala (motivo por el cual se construyó un sistema ferroviario regional), la explotación de la raíz del zacatón y la producción de carbón de encino. Dichas actividades marcaron el inicio de un periodo acelerado de expoliación de los recursos forestales en la región. La hacienda funcionaba además como casa prestamista y obtenía ciertos recursos por la venta de derechos de uso del agua de los ríos que la cruzaban a la Compañía Minera del Rincón (Chevalier, 1985).

\footnotetext{
${ }^{2}$ Las cargas, eran equivalentes al peso que un animal (caballo o asno) pudiese aguantar; se estima que el peso de una carga oscilaba entre 90 a $115 \mathrm{~kg}$, repartidos en dos costales para equilibrar el peso en el animal (Velázquez y Pérez, 1953).
} 


\section{Cuadro 1 \\ Producción anual de La Gavia}

\begin{tabular}{lrlr}
\hline \multicolumn{1}{r}{ Producto } & Cargas & Tipo de ganado & Cabezas \\
\hline Maíz & 2,000 & Vacuno & 4,500 \\
Trigo & 1,500 & Ovino & 1,200 \\
Cebada & 2,000 & Mular & 150 \\
Haba & 100 & Asnal & 80 \\
& & Porcino & 200 \\
\hline
\end{tabular}

Fuente: Villada (1893: 629); Sagarpa (2001: 118).

Hacia 1915 se promulgó a nivel nacional la ley que declaraba la reconstitución y dotación de tierras a los ejidos. Doña Dolores García Pimentel, dueña de la Gavia, previendo la inminente afectación a sus tierras, repartió una porción de su heredad entre 500 de sus peones. Estos pasaron a ser pequeños propietarios y fueron reconocidos años más tarde durante la visita del presidente Lázaro Cárdenas a la región en 1936, cuando ya había fallecido el último propietario de la hacienda, don Antonio Riba y Cervantes. Las reformas producidas por el movimiento agrario marcaron el inicio de la cesión de terrenos y la creación de ejidos. En pocos años los territorios de la hacienda se vieron reducidos a 222 hectáreas (GómezCarmona, 2000).

El proceso de desmembramiento de la hacienda no estuvo libre de conflictos. Los campesinos sin tierra agraristas pugnaban por el reparto y creación de ejidos y se enfrentaban a los antiguos rentistas o fraccionistas que, desde 1913, habían comenzado a comprar en abonos parte de las tierras de la hacienda (Martínez-Saldaña, 1983). Finalmente, poco más de 64,000 hectáreas de La Gavia se fraccionaron en 61 ejidos por dotación de tierras y 20 más por ampliación.

Al tiempo que se daba el reparto agrario en la región del Nevado de Toluca, el presidente Lázaro Cárdenas promovió, como parte de su visita en 1936, la promulgación del decreto de creación del Parque Nacional Nevado de Toluca. Así, los terrenos ejidales expropiados a la hacienda la Gavia conformaron el área natural protegida, quedando en su interior el cráter del volcán, todo lo cual se constituyó como una reserva forestal nacional a partir de la cota de los 3,000 msnm (GEM, 1990).

Los terrenos que constituyen el parque nacional abarcan algunas zonas ejidales de los municipios de Zinacantepec, Villa Guerrero, Texcaltitlán, Temascaltepec, Amanalco, Villa Victoria, Almoloya de Juárez, Toluca, Calimaya, Coatepec Harinas y Tenango del Valle. Entre las motivaciones para crear el área protegida destacaba la necesidad de conservar las con- 
diciones naturales de alta montańa, protegiéndola de la degradación y con la finalidad de asegurar el abastecimiento de agua necesaria para la agricultura y la industria.

\section{La Peńuela, un pueblo de alta montańa}

La Peñuela es una pequeña localidad ubicada en la zona limítrofe occidental del Parque Nacional Nevado de Toluca, a una altitud promedio de 3,040 msnm (GEM, 1990) (figura 1). El pueblo se localiza entre los ríos La Hortaliza y Pichontagüi y próximo a tres manantiales permanentes (figura 2). La existencia de suelos ricos en materia orgánica y la abundancia de recursos hidrológicos en la región han permitido el desarrollo de la agricultura de riego.

\section{Figura 1}

\section{Ubicación de La Peńuela en el Parque Nacional Nevado de Toluca}

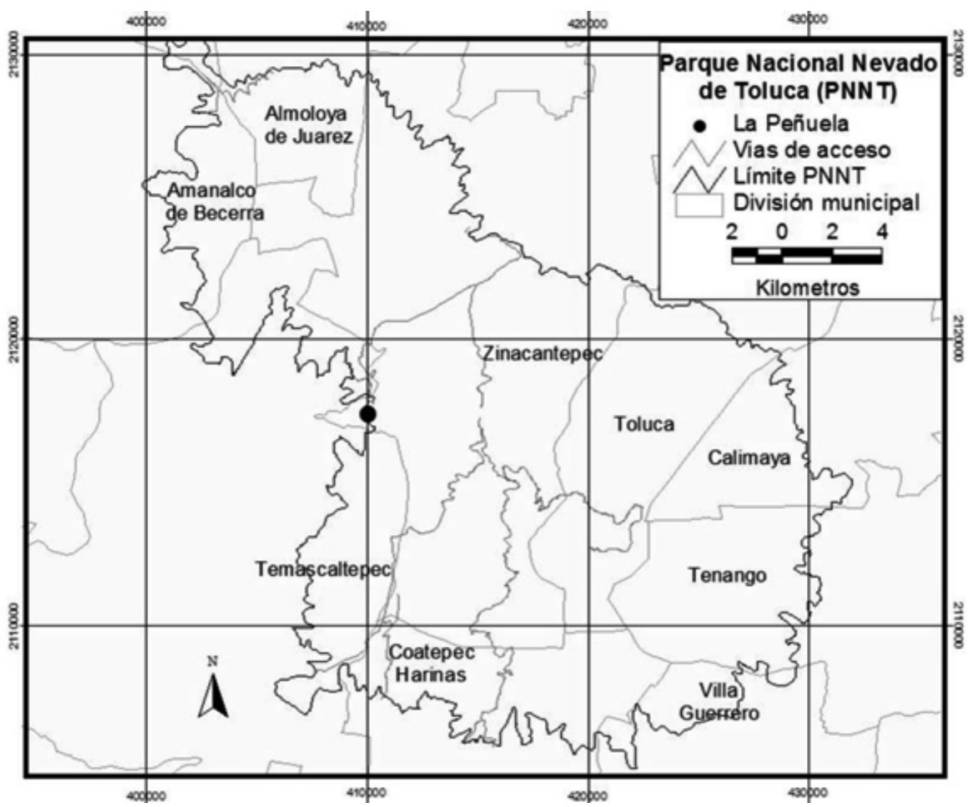

Fuente: elaboración propia.

De acuerdo con los pobladores de la región, los fundadores de la ranchería La Peñuela fueron los Felipes (Felipe Mendoza y Felipe García), posiblemente originarios de San Miguel Oxtotilpan. Sin embargo, existen registros de que los otomíes y matlazincas formaban parte de tribus nahuas, y una porción de estas etnias se estableció en el extenso y elevado altiplano, entre ríos y lagos, y en las faldas del Nevado de Toluca en los años 1200 


\section{Figura 2}

\section{La Peńuela: los manantiales y sus zonas de distribución de agua}

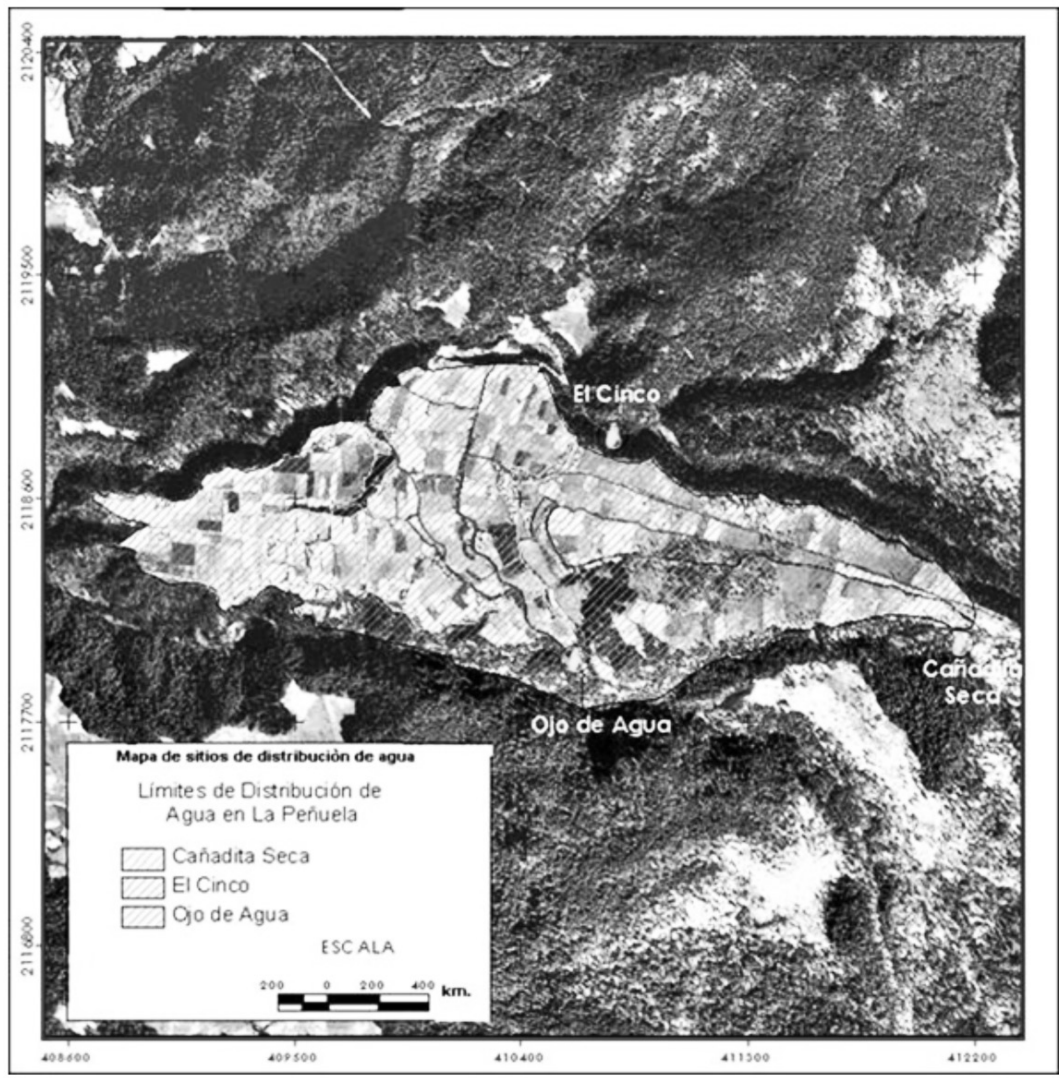

Fuente: elaboración propia.

(Pérez-Alvirde, 1999; Albarrán, 1991). Hacia 1903, Felipe García obtuvo en arrendamiento y, exclusivamente para la crianza de ganado vacuno, los territorios en torno a un ojo de agua custodiado por una gran peña (de ahí el origen de su nombre). Meses más tarde llegó al lugar su yerno, el señor Felipe Mendoza, con la finalidad de ayudarle con el cuidado del ganado. Hacia el año de 1906 las familias de Felipe García y Felipe Mendoza se mudaron a la ranchería para establecerse en proximidad al ojo de agua, dando origen a un lento proceso de poblamiento que, inevitablemente, conduciría a la adopción de nuevas actividades productivas.

Los destinos de la comunidad de La Peñuela se vieron marcados por dos sucesos prácticamente simultáneos. El 25 de enero de 1936 se decretó la creación del Parque Nacional Nevado de Toluca con miras a conservar la flora y fauna comarcanas, incluyendo en dicho decreto los terri- 
torios que ocupaba la ranchería. Meses más tarde, el primero de julio se publicó en el Diario Oficial de la Federación la dotación de 432 ha para la creación del ejido de La Peñuela, de las cuales 116 hectáreas serían destinadas para el caserío de la comunidad y el resto de los terrenos de agostadero para la cría del ganado (RAN, 1936). Al año siguiente, con la finalidad de conciliar los intereses de conservación y de desarrollo comunal se publicó el 19 de febrero de 1937 en el Diario Oficial de la Federación una modificación al decreto de creación del área natural protegida contemplando la creación de una reserva forestal para la explotación racional del bosque bajo la supervisión gubernamental. Así, el 18 de mayo de 1937 se ejecutó la acción de dotación de tierras a 35 ejidatarios de La Peñuela, quedando establecido con ello el derrotero que seguiría el uso y aprovechamiento del territorio en la región.

Años más tarde, hacia el 12 de febrero de 1952 se ejecutó la acción de dotación de 220 hectáreas para la ampliación del ejido que fue publicada por resolución presidencial en 1951, de las cuales 120 eran de cultivos de riego, 80 de monte y 20 de ocupación de barrancas. Los vecinos de la comunidad ocuparon por cerca de tres años de forma ilegal esta superficie ampliada hasta que se les concedió la ampliación del ejido (RAN, 1952). De esta manera, la superficie total del ejido llegó a las 652 hectáreas en terrenos dentro y fuera del área natural protegida.

El primer dato censal formal sobre población total se remonta a 1940, cuando la localidad contaba con 209 habitantes (cuadro 2). Para la segunda mitad del siglo xx la población se mantuvo en torno al medio millar de habitantes con un ligero repunte en la primera década del siglo xxI.

Si bien los censos reflejan un incremento relativamente constante de la población durante las primeras décadas de fundación de la localidad, el incremento poblacional de 1940 al 2010 fue de 32\%, es decir, hubo un incremento de 446 personas considerando el proceso evolutivo de las personas (INEGI, 1940-2010). Los habitantes del pueblo afirman que el crecimiento de la población se vio afectado por altas tasas de mortalidad infantil consecuencia de la pulmonía, la intoxicación por inhalación del humo de los hogares y la varicela (Anastacio-Martínez, 2011). Estos problemas estaban asociados a la pobreza extrema y los rigores del clima en la alta montańa. De acuerdo con los conteos y censos de población y vivienda publicados de 1940 a 1960, el número de mujeres era mayor al de los hombres; hacia el año de 1970 no se tiene registro por sexo, mientras del año 1980 al 2010 el incremento en el número de hombres es mayor. 


\section{Cuadro 2}

Población de La Peñuela

\begin{tabular}{lccc}
\hline \multicolumn{1}{c}{ Año } & Total & Hombres & Mujeres \\
\hline 1940 & 209 & 95 & 114 \\
1950 & 289 & 127 & 162 \\
1960 & 261 & 130 & 131 \\
1970 & 290 & nd & nd \\
1980 & 358 & 201 & 157 \\
1990 & 457 & 241 & 216 \\
1995 & 530 & 284 & 246 \\
2000 & 577 & 304 & 273 \\
2005 & 528 & 275 & 253 \\
2010 & 655 & 332 & 323 \\
\hline
\end{tabular}

nd = no determinado.

Fuente: Censo General de Población y Censo de Población y Vivienda, INEgi publicado de 1940 a 2010 .

De acuerdo con Abasolo (2006), hacia finales del siglo xIx la actividad económica en los pueblos de alta montaña giraba en torno a la explotación de los recursos del bosque, lo que incluía la extracción de madera, raíz de zacatón, recolección y caza. Las comunidades enfrentaban limitantes impuestas por el régimen de subsistencia, los factores climáticos y los elementos de un entorno hostil. El incremento de riesgos ambientales y económicos condujo a los habitantes a seguir estrategias que propiciaron la deforestación, lo que conllevó a la trasformación de sistemas forestales típicos a sistemas agropecuarios y a un cambio de los modos de vida, como lo mencionan los habitantes de la comunidad "el bosque lucía tupido, lleno de árboles, razón por la cual sólo los hombres eran quienes entraban al bosque a sacar la leña necesaria, lo cual cambió cuando empezamos a sembrar las tierras".

\subsection{El desarrollo de las actividades agrícolas y ganaderas}

La actividad agropecuaria en La Peńuela ha pasado por diversos periodos, durante el establecimiento de la ranchería que corresponde con la primera década del siglo xx, la actividad económica se centraba exclusivamente en la crianza extensiva de ganado vacuno. El periodo entre 1920 y hasta finales de los cuarenta se caracterizó por el predominio de la actividad pecuaria pero ya con la incorporación de actividades agrícolas de subsistencia por medio del cultivo del maíz. Hacia los ańos cincuenta se dio 
una transición hacia el cultivo de la papa y la sustitución de la cebada por el trigo como cultivo forrajero. Desde principios de los setenta y hasta finales de los noventa se presentó un cambio en la actividad pecuaria hacia la crianza extensiva de ganado ovino, así como la diversificación de los cultivos y la mecanización de la agricultura. Finalmente, durante la primera década del siglo xxi disminuyó radicalmente la producción pecuaria, prevaleciendo la agricultura comercial principalmente de papa y flores de ornato.

\subsection{El establecimiento}

El establecimiento de la ranchería a principios de siglo xx se basó en la crianza extensiva de ganado vacuno que solía venderse en los mercados regionales de Toluca y Zinacantepec. El ganado era alimentado exclusivamente por pastos y herbáceas nativas que crecían en las zonas de pastoreo y su crianza dependía en buena medida de la abundante disponibilidad de agua del manantial que brotaba en la base de la Peñuela. El pastoreo de los animales se realizaba de manera trashumante aunque por las noches los animales eran encerrados en corrales con la finalidad de prevenir el abigeato y el ataque de lobos y coyotes que habitaban las zonas boscosas. En esta etapa, dada la poca población existente en la zona, no había una extracción desmedida de recursos maderables que se destinaban plenamente a la subsistencia. Entre los elementos utilizados destacan la madera verde para la protección de los animales domésticos, leña muerta para los hogares y los hongos comestibles (clavitos y orejas principalmente). Adicionalmente se practicaba la cacería de conejos y venados. El principal impacto negativo sobre el medio ambiente derivaba de una creciente actividad pecuaria extensiva.

\subsection{Periodo de predominio de la actividad pecuaria e inicios de la agricultura}

Hacia los años veinte, con la promulgación de la ley que declaraba la reconstitución y dotación de tierras a los ejidos, con la pérdida de influencia de los propietarios de la hacienda y con el incremento poblacional que había experimentado la ranchería, se dio paso a la apertura de zonas de cultivo destinadas fundamentalmente a la producción de maíz para el autoconsumo. La crianza de ganado vacuno continuaba siendo la única actividad productiva comercial y por ende se trataba de una comunidad de pastores que durante el día llevaban los hatos ganaderos a las zonas de pastoreo y por las noches los estabulaban para evitar el abigeato. Dadas las condiciones ambientales y orográficas, así como las rudimentarias 
técnicas de cultivo, ${ }^{3}$ se obtenían rendimientos muy bajos de un maíz de mala calidad. Si bien durante esta década, como resultado de la revolución mexicana, el Estado de México y el resto del país transitaron hacia la producción de granos para su exportación (principalmente maíz, cebada y, en menor escala, avena) (Martínez-Saldaña, 1983), esto no incidió significativamente en la producción agrícola local.

Hacia principios de los años veinte se inició la apertura de canales de riego con la finalidad de garantizar la producción del grano, cuando aún la actividad agrícola era incipiente. Los canales servían fundamentalmente para llevar agua a las viviendas de la comunidad y para garantizar el suministro para los animales. De acuerdo con los lugareños, desde su inicio la organización en torno al uso y manejo del agua fue comunal.

Durante el periodo de los años treinta y cuarenta, además de la crianza del ganado vacuno y del cultivo de maíz para autoconsumo, se introdujo el cultivo de cebada con la finalidad de satisfacer la demanda de la industria cervecera que se había establecido en el Valle de Toluca. Sin embargo, la calidad de la cebada cultivada no satisfacía los requerimientos para la fabricación de cerveza por lo que la producción se utilizaba como complemento alimenticio del ganado. ${ }^{4}$ Hacia finales de este periodo la extensión de las zonas destinadas al cultivo de maíz tendió a disminuir ya que, a pesar de contar con riego ${ }^{5}$ y del uso de abono animal en las parcelas, ${ }^{6}$ la producción se vio seriamente afectada por los efectos del clima adverso. Esta situación concuerda con Rivera-Herrejón (2007), quien afirma que durante la década de los cuarenta las zonas rurales del Estado de México se caracterizaban principalmente por la actividad agrícola y ganadera que permitía la subsistencia de sus habitantes. ${ }^{7}$

${ }^{3}$ El uso del azadón como principal herramienta para el manejo de la tierra en La Peñuela prevaleció hasta fines de la década de los cuarenta, utilizándose para la remoción de tierra, la siembra, la cosecha, la formación de surcos, etc. Se estima que con la utilización del azadón, un peón podía abarcar unos 200 a 300 metros diarios de tierra. En cambio, con el arado el tiempo invertido en el cultivo, así como el número de brazos requeridos para cultivar la misma superficie se reducía una tercera parte (Menegus-Bornemann, 1998).

${ }^{4}$ Mendoza-Vargas (1979) afirma que la cebada era un cultivo ampliamente difundido en el valle de México, propio de tierras pobres y de áreas de mal temporal, y se utilizaba generalmente para el forraje de los animales de trabajo.

${ }^{5}$ Desde el inicio de la actividad agrícola en la localidad se abrieron los primeros canales de riego a partir del Ojo de Agua.

${ }^{6}$ De acuerdo con Rivera-Herrejón (2007), durante la década de los cuarenta, la producción agrícola de la entidad creció muy lentamente, sobre todo por el comportamiento del maíz, debido a que se presentó un aumento significativo de la producción de trigo, papa y frijol. Debido a que los agricultores del estado aplicaban abono animal para fertilizar los campos, la escasez de este insumo impedía recuperar suficientemente la fertilidad de los suelos.

${ }^{7}$ El estancamiento de la producción agrícola junto con el sostenido aumento de la población rural se había traducido en un notorio incremento de la pobreza en el campo, hecho que incluso consignaban los documentos oficiales (Codagem, 1979). 
Otro de los elementos que influyó para que la ganadería y las actividades agrícolas tuvieran un mayor auge en La Peñuela fue la declaración del decreto de la zona como Parque Nacional. De esta manera los habitantes de la localidad enfrentaban la prohibición de la caza de animales y la extracción de recursos forestales. La extracción de madera muerta para el autoconsumo era la única actividad extractiva permitida y los montos utilizados dependían en buena medida del incremento de la población. Por tanto, la ausencia de condiciones favorables en dichas localidades provocó que la ganadería extensiva fuera la única actividad que pudiera contribuir a la subsistencia de la comunidad.

\section{Periodo de transición hacia la actividad agrícola comercial}

La década de los cincuenta se caracterizó por la transición de la actividad pecuaria hacia el desarrollo de la agricultura comercial, principalmente del cultivo de papa. El pastoreo del ganado vacuno pasó a ser una actividad secundaria. Si bien el cultivo de la papa se destinaba al autoconsumo, los excedentes de la producción se vendían en los mercados de Toluca, Zinacantepec y México. De acuerdo con informantes de la localidad, este cultivo fue introducido por personas que habían migrado a la ciudad de Toluca y que regresaron al pueblo con los conocimientos básicos para su manejo. Los primeros intentos se realizaron utilizando abono natural. ${ }^{8}$ Una vez observado que la papa resistía los rigores del clima de la montaña y contando con agua suficiente para riego, ${ }^{9}$ el cultivo se consolidó como el más importante en la región.

En este contexto, ante las mayores ganancias derivadas de la comercialización de la papa, la producción ganadera decayó sensiblemente y con ello el cultivo de cebada que pasó a ser reemplazado por el cultivo de trigo como forraje. El maíz de mala calidad se continuaba cultivando para el consumo humano. Durante esta época el arado de la tierra se realizaba mediante tracción animal (principalmente caballos y mulas) con rastras de madera (Anastacio-Martínez, 2011).

\footnotetext{
${ }^{8} \mathrm{El}$ abono natural fue utilizado desde el inicio de la agricultura en las zonas rurales como fertilizante, como consecuencia del poco capital que permitiera la compra de fertilizantes comerciales y el conocimiento necesario sobre su aplicación. En la década de los cincuenta se inició propiamente la difusión en el uso de los fertilizantes químicos en el Estado de México. En esos años el crecimiento estimado del consumo de fertilizantes fue muy elevado; por otro lado, no existía entre los agricultores el conocimiento necesario para hacer un adecuado uso del fertilizante comercial (RiveraHerrejón, 2007).

${ }^{9}$ El riego de los cultivos se realiza de forma escalonada, iniciando por los terrenos más cercanos a los canales y alternando el riego entre el día y la noche.
} 


\section{Periodo de consolidación del cultivo de la papa y diversificación de la actividad agrícola}

Durante la década de los sesenta y buena parte de los setenta se presentó el auge del cultivo de la papa y apareció la rotación de cultivos, convirtiendo a la agricultura en el eje de desarrollo económico de la comunidad. Así, se adoptó la siembra de haba para el autoconsumo, en rotación con la avena y la papa y prácticamente desaparece el cultivo de maíz, lo cual pudo atribuirse no solo a la mala calidad del grano cocechado en la localidad sino al sensible aumento de la oferta de este producto a nivel estatal. ${ }^{10}$ El pastoreo de ganado bovino no es olvidado y forma parte de una actividad económica complementaria y, gracias a los intercambios comerciales de los pastores, se introduce la crianza y pastoreo de borregos destinados a la venta como animales vivos.

Hacia la década de los ochenta la agricultura de riego ${ }^{11}$ (principalmente el cultivo de la papa) siguió siendo la principal actividad económica de la localidad y la crianza de borregos se consolidó como la segunda actividad más importante. Se continuó con el cultivo de haba para autoconsumo y venta, así como con la producción de quelites. La avena se cultivaba como complemento alimenticio de los animales. De acuerdo con Rivera-Herrejón (2007), estos años se caracterizaron por la reducción de la producción agrícola del estado, principalmente por el descenso de la producción de maíz. Esto, sin embargo, parece no haber afectado la actividad agrícola local que había abandonado ya el cultivo de este grano básico.

En 1990 llegó a la comunidad el primer tractor que se alquilaba para el manejo de las parcelas. A partir de esa fecha algunos productores locales han venido adquiriendo maquinaria mediante los programas de apoyo del estado al fomento agrícola. Por otro lado, cabe destacar que la compra de tractores ha estado muy vinculada con aquellos productores que cuentan con extensiones de tierra significativas y que se dedican al cultivo comercial de la papa. Los años noventa marcan también la intro-

${ }^{10}$ En la década de los sesenta, $97 \%$ del territorio mexiquense se dedicaba al cultivo de los ocho principales productos; asimismo, aumentó el uso de los fertilizantes inorgánicos que beneficiaron presumiblemente a la superficie cultivada de maíz. En 1965 la producción agrícola ascendió, registrando en la producción del maíz una gran expansión de la superficie cosechada; otros productos con crecimiento en el periodo fueron la papa, la cebada grano, el frijol y el haba (Codagem, 1979). La situación local reflejaba lo que venía ocurriendo a nivel estatal, es decir, la estructura del valor de la producción se modificó a favor de los productos como la alfalfa, el frijol, la papa y la avena grano, mientras que disminuyó la producción del maíz y la cebada (Rivera-Herrejón, 2007).

${ }^{11}$ Si bien hasta la década de los ochenta el agua proveniente del Ojo de Agua fluía libremente por el cauce del río, fue en esta década cuando se construyó una pequeña represa que permitió encausar las aguas del manantial por los canales, con un gasto de 800 litros de agua por segundo. Esto posibilitó además la colocación de tuberías para el abastecimiento de agua potable de las viviendas. Ante el crecimiento de la localidad, los habitantes de zonas más alejadas tuvieron que recurrir a nuevas fuentes de suministro de agua. 
ducción de otros cultivos como el chícharo y el rábano que probaron ser resistentes a las condiciones climáticas locales. ${ }^{12}$

La situación de los recursos maderables sufrió importantes cambios. Con el paso del tiempo, la gente de la comunidad que había respetado las prohibiciones que el decreto de creación del parque nacional, incrementa su actividad extractiva. En efecto, a partir de los sesenta, con el crecimiento de la población y la baja en la productividad agrícola, la gente emprendió una extracción a gran escala de leña verde con la finalidad de comercializarla en los mercados regionales. El argumento central para esta expoliación de recursos radica en que la comunidad era muy pobre y necesitaban del dinero para comer; los habitantes de la comunidad mencionan que "durante la madrugada extrañaba a la gente escuchar el ruido de los animales o del caer de los árboles y, conforme pasó el tiempo, los ruidos se hicieron más continuos". Como consecuencia, la explotación de la madera no sólo se dio a nivel local sino que habitantes ajenos a la comunidad también la explotaban sin ningún control.

En esta época un grupo de personas recurrió a las autoridades de Zinacantepec para reportar las extracciones ilegales. A raíz de ello, las instituciones gubernamentales federales entraron en las zonas afectadas y organizaron a la gente de la comunidad para cuidar el bosque durante las noches y hacer seńales de alerta cuando los taladores aparecieran. Como resultado de esto, un grupo de personas de Acahualco, municipio de Zinacantepec fueron detenidas por la extracción ilegal de leña, y la gente de la comunidad recibió capacitación para la reforestación de su bosque.

Durante la década de los noventa se comenzaron a visualizar las primeras señales de la reforestación y desde ese momento en la comunidad no se permite la comercialización de la leña pero sí la extracción de madera para el consumo en los hogares; actualmente dicho uso se da en $100 \%$ en las casas de la comunidad ya que la gente no ve la necesidad de comprar gas.

\section{Periodo de diversificación de la agricultura comercial}

Durante la primera década del siglo xxi ha prevalecido en la localidad el cultivo de la papa como motor de la agricultura. En los últimos años ha venido proliferando el cultivo de flores a cielo abierto o en invernadero. Esta actividad ha sido promovida por medieros $^{13}$ provenientes de la región

${ }^{12}$ De 1991 a 2004 la producción agrícola del estado tuvo amplias fluctuaciones que dificultan identificar una tendencia. Los cultivos tradicionales de papa, chícharo y haba perdieron participación en la superficie cosechada, mientras que otras hortalizas, como el tomate verde, zanahoria y el elote ganaron participación en la producción y la superficie cosechada.

${ }^{13}$ Los medieros son personas que ofrecen al agricultor y dueńo de la tierra la mitad de las ganancias que se obtienen por la venta de los productos; es decir, la inversión empieza desde la siembra, 
de Villa Guerrero y que invierten en la producción de flores de ornato como la nube, ${ }^{14} \mathrm{el}$ cempazúchitl,,${ }^{15}$ la margarita y el crisantemo, ${ }^{16}$ así como de plántulas para su venta en los invernaderos de aquella importante región florícola (Conanp, 2013b). Hacia el año 2007, el cultivo de papa abarcaba $73 \%$ de la superficie agrícola, mientras $1.7 \%$ correspondía al cultivo de avena forrajera (cuadro 3). Se estima que hacia el ańo 2009 la cubierta forestal cubría más de $70 \%$ de la superficie del ejido, mientras una mínima parte era ocupada por los cuerpos de agua, asentamientos humanos, caminos, etc. (cuadro 4).

\section{Cuadro 3}

\section{Producción agrícola y pecuaria en La Peńuela}

\begin{tabular}{lc}
\hline \multicolumn{1}{c}{ Actividad } & Porcentaje \\
\hline Cultivo de leguminosas & 7 \\
Cultivo de papa & 73 \\
Cultivo de avena forrajera & 1.70 \\
Cultivo de hortalizas & 5 \\
Explotación de ovinos & 1.70 \\
Explotación de equinos & 11 \\
Explotación de bovinos & 0.60 \\
Total & 100 \\
\hline
\end{tabular}

Fuente: SCIGA (2007).

en la compra de la semilla, fertilizante y mano de obra y, una vez que se cosecha el producto y se vende, las ganancias obtenidas se aprovechan por partes iguales.

${ }^{14} \mathrm{El}$ primer cultivo de flor que se implementó en la localidad fue la nube que se siembra en los meses de febrero, abril y octubre; los medieros la comercializan en las centrales de abastos de Toluca y del Distrito Federal.

${ }^{15}$ El cempazúchitl se empezó a cultivar en el año 2000, sembrándose en los meses de septiembre y octubre, programando su fecha de corte para las celebraciones de difuntos. Se trata de un cultivo para venta pero también de autoconsumo.

${ }^{16}$ En 2003 se inició el cultivo en invernadero de margarita. La planta se siembra en el mes de marzo y su destino es la comercialización de la flor en los mismos sitios de distribución que la nube. El cultivo del crisantemo se inició en 2008 y suele realizarse en los meses de junio y julio. 


\section{Cuadro 4}

\section{Ocupación de la tierra ejido de La Peńuela 2009}

\begin{tabular}{lrc}
\hline \multicolumn{1}{c}{ Ocupación } & Porcentaje & Superficie (has.) \\
\hline Forestal & $78.37 \%$ & 511 \\
Agrícola & $16.41 \%$ & 107 \\
Pastizal & $4.14 \%$ & 27 \\
Otros usos & $1.08 \%$ & 7 \\
Total & $100.00 \%$ & 652 \\
\hline
\end{tabular}

Otros usos: cuerpos de agua, barrancas, asentamientos humanos, caminos, áreas antrópicas y áreas desprovistas de vegetación.

Fuente: elaboración propia a partir del mapa de ocupación del suelo 2009 del Parque Nacional Nevado de Toluca.

Aún cuando las actividades agrícolas tienen un auge económico importante, los recursos forestales no dejan de recibir presiones de explotación principalmente en lo referente a los recursos no maderables como son los hongos (Vallejo et al., 2013). Año con año, durante la época de lluvias se da la visita constante de señoras provenientes de Acahualco, en el municipio de San Miguel Zinacantepec y otros poblados cercanos que recorren los bosques circunvecinos en busca de los hongos. Este importante recurso natural, como los clavitos, orejas, gachupines, patas de pájaro y cornetas, se empezó a comercializar entre 1990-2000 y las estimaciones sugieren que en promedio se pueden extraer hasta $280 \mathrm{~kg}$ de hongos entre junio y octubre. Otro recurso que se extrae de forma ilegal es la tierra de monte. De acuerdo con la Subprocuraduría de Recursos Naturales y la Dirección General de Inspección y Vigilancia Forestal, el Estado de México y en particular la Sierra del Nevado de Toluca forman parte de las zonas críticas forestales; la Peñuela es parte de estas zonas, lo que constituye un elemento adicional que tienen los recursos forestales y que han influido en los modos de vida de los habitantes (Profepa, 2011).

\section{Conclusiones}

El proceso histórico que ha tenido La Peńuela indica que las actividades agropecuarias han influido de manera paralela en la explotación de los recursos forestales. La ganadería extensiva en sus inicios sólo utilizaba estos recursos con fines de subsistencia, pero la agricultura, al proveer de beneficios económicos, desplazó a la ganadería como actividad principal sin dejar a un lado la explotación de los recursos forestales. La introducción de este tipo de cultivos comerciales hizo que el agua tuviera mayor 
importancia para su buen desempeño pero también provocó un incremento de la presión en la utilización de este recurso.

Aún cuando los cultivos de flor han tenido éxito en su producción dada la disponibilidad de agua, los dueños de los terrenos no están dispuestos a sustituir los cultivos de papa por los de flor y es posible encontrar cultivos del tubérculo en la infraestructura que corresponde a las flores. El impedimento en la sustitución de cultivos se ve influenciada por la falta de conocimiento de los habitantes respecto al ciclo agrícola de las plantas de ornato, así como de su comercialización.

La transformación con relación a los recursos maderables y no maderables se dio con una tendencia hacia una comercialización temporal, pero a su vez ha ocasionado el deterioro ambiental que ha conllevado a la escasez de hongos, a la tala clandestina y a la extracción de leña muerta para ser comercializada en lugares lejanos al parque.

Las herramientas de análisis utilizadas aquí sirvieron para identificar las percepciones de la comunidad en este proceso histórico, mientras que las tendencias a futuro sugieren que la incidencia o implantación de nuevos cultivos están enfocados al aporte económico que convenga a la gente de la comunidad y a la disponibilidad del agua, ya que es el elemento clave que rige el desarrollo de esta comunidad de alta montaña.

El cambio, en la ocupación del suelo que sufrió el parque desde la existencia de La Gavia, y los acontecimientos que siguieron, han constituido el conjunto de procesos de incidencia ambiental que han degradado una de las principales zonas de recarga acuífera del Estado de México. Esto representa a la vez un caso de estudio de los pueblos que se desarrollan en la alta montańa y de los medios de subsistencia que implementan, dadas las condiciones ambientales y geográficas.

\section{Bibliografía}

Abasolo-Palacio, Víctor Enrique (2006), "Entre el cielo y la tierra: raíces, un pueblo de la alta montaña en el Estado de México", tesis de doctorado, Universidad Iberoamericana, México.

Albarrán de Rivera, María Estela (1991), Hacienda La Gavía, breve relato de su historia Estado de México, Promotora La Gavia Toluca.

AgN (Archivo General de la Nación) (1668), Tierras: La Gavia, v. 2795 exp., fs. 10-53.

Agnem (Archivo General de Notarias del Estado de México) (1663), Expedientes de La Gavia, c 23, 15, fs. 89-90. 
Chevalier, Francois (1985), La formación de latifundios en México, FCE, México.

Codagem (Comisión Coordinadora para el Desarrollo Agrícola y Ganadero del Estado de México) (1979), Evolución Agrícola del Estado de México, 1940-1975, Codagem, Toluca.

Conanp (Comisión Nacional de Áreas Naturales Protegidas) (2013a), "Estudio previo justificativo para la modificación de la declaratoria del Parque Nacional Nevado de Toluca”, <http://www.toluca. gob.mx/sites/default/files/Nevado/Nevado\%20de\%20Toluca $\% 20$ 2013.pdf>, 24 de enero de 2014.

Conanp (Comisión Nacional de Áreas Naturales Protegidas) (2013b), "Borrador del programa de manejo del ApfF Nevado de Toluca, para consulta pública en cumplimento a lo previsto por los artículos 65 de la Ley General de Equilibrio Ecológico y la protección al ambiente y 73 de su reglamento en materia de áreas naturales protegidas, <http://www.conanp.gob.mx/anp/consulta/BORRADOR\%20PM\%20NEVADO\%20DE\%20TOLUCA-311013. pdf>, 24 de enero de 2014.

GEM (Gobierno del Estado de México) (1990), Programa de manejo del Parque Nacional Nevado de Toluca, Secretaría de Ecología-Universidad Autónoma del Estado de México, Toluca.

Gómez-Carmona, Gabriel (2000), "Hacienda La Gavia, siglo XvI-xx. Su historia y evolución arquitectónica”, tesis de licenciatura, Universidad Autónoma del Estado de México, Toluca.

Guzmán-Urbiola, Xavier (1998), Hacienda La Gavia, Promotora La Gavia, México.

Hernández-Rodríguez, Rosaura (2005), Zinacantepec en la época prehispánica siglos XV y XVI, El Colegio Mexiquense, A.C., Zinacantepec.

INEGI (Instituto Nacional de Estadística y Geografía) (1940-2010) Censo de Población y Vivienda, Instituto Nacional de Estadística y Geografía, México.

Martínez-Saldaña, Tomás (1983), "Historia de la agricultura en México", ponencia presentada en el III Taller Latinoamericano Prevención 
de Riesgos en el Uso de Plaguicidas, Instituto Nacional de Investigaciones sobre Recursos Bióticos, 1-6 de diciembre, Xalapa.

Mendoza-Vargas, Gilberto (1979), Evolución agricola en el Estado de México, 1940-1975, Codagem-Gobierno del Estado de México, Toluca.

Menegus-Bornemann, Margarita (1998), "Haciendas y comunidades en el valle de Toluca, siglos Xviı y Xviıı”, en Teresa Jarquín y Manuel Miño Grijalva (coords.), Historia General del Estado de México. La época virreinal, El Colegio Mexiquense, A.C., Zinacantepec, pp. 295-311.

Montes de Oca, Elvia (1998), "Las haciendas”, en Teresa Jarquín y Manuel Miño (coords.), Historia General del Estado de México. Independencia, Reforma e Imperio, El Colegio Mexiquense, A.C., Zinacantepec, pp. 7-18.

Pérez-Alvirde, Rosalío (1999), Zinacantepec. Monografía municipal, Instituto Mexiquense de Cultura-Asociación Mexiquense de Cronistas Municipales, Toluca.

Profepa (Procuraduría Federal de Protección al Ambiente) (2011), “Zonas críticas de extracción de recursos maderables", <http://profepa. $\mathrm{mx} /$ innovaportal/v/3489/1/mx/zonas_criticas_forestales. html>, 26 de julio de 2011.

RAN (Registro Agrario Nacional) (1936), "Núcleo Agrario: ejido La Peñuela”, Serie documental, Programa de Certificación de Derechos Ejidales y Titulación de Solares, expediente 42/700.

RAN (Registro Agrario Nacional) (1952), "Núcleo Agrario: ejido La Penuela”, Serie documental, , Resolución presidencial de ampliación de hectáreas, expediente 42/700.

Rivera-Herrejón, Gladys (2007), El desarrollo agrícola en el Estado de México 1940-2004, Universidad Autónoma del Estado de México, Toluca.

Rosenzweig, Fernando, Rosaura Hernández, María Teresa Jarquín y Manuel Miño Grijalva (1987), Breve Historia del Estado de México, El Colegio Mexiquense, A.C., Zinacantepec. 
Sagarpa (Secretaría de Agricultura, Ganadería, Desarrollo Rural, Pesca y Alimentación) (2001), Producción agrícola y pecuaria, datos por entidad, Anuario Estadístico de México, Inegi, México.

SCIGA (Sistema de Consulta de Información Geoestadística Agropecuaria) (2007), Censo agropecuario 2007, <http://gaia.inegi.org.mx/ sciga/viewer.html>, 28 de julio de 2011.

Velázquez, Gustavo y Ramón Pérez (1953), La Gavia, biografía de una hacienda mexicana, Editores Asociados, México.

Vallejo, Blanca, Maribel Osorio, Irma Ramírez, Gabino Nava y Sergio Maass (2013), "Análisis social sobre los habitantes de la comunidad de La Peñuela, Parque Nacional Nevado de Toluca, México”, Estudios y perspectivas de género, 22 (3), Centro de investigaciones y estudios turísticos, Buenos Aires, pp. 425-449.

Villada, José Vicente (1893), "Memoria de la Administración Pública del Estado de México”, presentada a la XV Legislatura de 1889 a 1893, Toluca, pp. 629-630.

Entrevista:

Anastacio-Martínez, Nancy Diana, entrevista realizada a Bacilizo Mata García, La Peñuela, Parque Nacional Nevado de Toluca, Zinacantepec, 15 de abril de 2011.

Recibido: 10 de octubre 2011. Reenviado: 27 de septiembre de 2012. Aceptado: 22 de octubre de 2012.

Nancy Diana Anastacio-Martínez. Es licenciada en geografía y maestra en ciencias agropecuarias y recursos naturales; su línea de investigación es el manejo y conservación de recursos naturales. Actualmente es estudiante de doctorado en ciencias agropecuarias y recursos naturales en el Instituto de Ciencias Agropecuarias y Rurales de la Universidad Autónoma del Estado de México.

Gabino Nava-Bernal. Es doctor en ciencias agropecuarias por la Universidad de East Anglia-Reino Unido. Investigador SNI nivel I con perfil deseable Promep. Su área de investigación es el manejo y conservación de 
recursos naturales. Recibió el Premio Estatal para la Conservación por el Gobierno del Estado de México y la Secretaría de Medio ambiente 2011. Entre sus últimas publicaciones destacan, en coautoría: "Participatory generation of sustainability indicators in a natural protected area of México", Ecological Indicators, 25, Elsevier, Seattle, pp. 1-9 (2013); en coautoría, "Effect of human disturbance on the structure and regeneration of forests in the Nevado de Toluca National Park", Journal of Forestry Research, 23(1), Northcast Forestry University-Springer, Berlin, pp. 39-44 (2012); en coautoría: "Estructura y regeneración en bosques tropicales de alta montańa: el caso del Parque Nacional Nevado de Toluca”, Bosques y árboles del trópico mexicano: estructura y crecimiento y usos, Prometeo editores, México, pp. 2-22 (2012); y "El líquido precioso, promotor de la vida. Formas de organización social en torno a un recurso", en Franco Maass (comp.), Monte Tlaloc II. La casa del Dios del Agua, Universidad Autónoma del Estado de México, México, 17 pp. (2012).

Sergio Franco-Maass. Es doctor en filosofía con especialidad en geografía por la Universidad de Alcalá de Henares, España. Miembro del SNI nivel II. Profesor investigador con perfil deseable Promep. Su área de investigación es manejo y conservación de recursos naturales. Entre sus últimas publicaciones destacan, en coautoría: "Análisis social sobre los habitantes de la comunidad de La Peñuela, Parque Nacional Nevado de Toluca, México", Estudios y Perspectivas en Turismo, 22 (3), Centro de Investigaciones y Estudios Turísticos de la Universidad Autónoma del Estado de México, Toluca, pp. 425-449 (2013); en coautoría: "Participatory generation of sustainability indicators in a natural protected area of México", Ecological Indicators, 25, Elsevier, Seattle, pp. 1-9 (2013); en coautoría: "Extracción de madera en el Parque Nacional Nevado de Toluca", Revista Mexicana de Ciencias Forestales, Instituto Nacional de Investigaciones Forestales, Agrícolas y pecuarias, México, pp. 81-90 (2012); en coautoría: "Genetic variability of sacred fir (Abies religiosa) in the Nevado de Toluca National Park", International Journal of Biodiversity and Conservation, Academic Journals, pp.130-136 (2012). 